\title{
Learning Behavior of Memristor-Based Neuromorphic Circuits in the Presence of Radiation
}

\author{
Sumedha Gandharava Dahl \\ Department of Electrical and \\ Computer Engineering \\ Boise State University \\ Boise, ID, 83725 USA \\ sumedhagandharava@boisestate.edu
}

\author{
Robert C. Ivans \\ Department of Electrical and \\ Computer Engineering \\ Boise State University \\ Boise, ID, 83725 USA \\ robertIvans@boisestate.edu
}

\author{
Kurtis D. Cantley \\ Department of Electrical and \\ Computer Engineering \\ Boise State University \\ Boise, ID, 83725 USA \\ kurtiscantley@boisestate.edu
}

\begin{abstract}
In this paper, a feed-forward spiking neural network with memristive synapses is designed to learn a spatio-temporal pattern representing the 25-pixel character ' $\mathrm{B}$ ' by separating correlated and uncorrelated afferents. The network uses spike-timing-dependent plasticity (STDP) learning behavior, which is implemented using biphasic neuron spikes. A $\mathrm{TiO}_{2}$ memristor non-linear drift model is used to simulate synaptic behavior in the neuromorphic circuit. The network uses a many-to-one topology with 25 pre-synaptic neurons (afferent) each connected to a memristive synapse and one postsynaptic neuron. The memristor model is modified to include the experimentally observed effect of state-altering radiation. During the learning process, irradiation of the memristors alters their conductance state, and the effect on circuit learning behavior is determined. Radiation is observed to generally increase the synaptic weight of the memristive devices, making the network connections more conductive and less stable. However, the network appears to relearn the pattern when radiation ceases but does take longer to resolve the correlation and pattern. Network recovery time is proportional to flux, intensity, and duration of the radiation. Further, at lower but continuous radiation exposure, (flux $1 \times 10^{10}$ $\mathrm{cm}^{-2} \mathrm{~s}^{-1}$ and below), the circuit resolves the pattern successfully for up to $100 \mathrm{~s}$.
\end{abstract}

\section{CCS CONCEPTS}

- Computer systems organization $\rightarrow$ Architectures $\rightarrow$ Other architectures $\rightarrow$ Neural networks $\bullet$ Hardware $\rightarrow$ Hardware test; Emerging technologies $\rightarrow$ Analysis and design of emerging devices and systems $\rightarrow$ Emerging architectures; Emerging Simulations

\footnotetext{
Permission to make digital or hard copies of all or part of this work for personal or classroom use is granted without fee provided that copies are not made or distributed for profit or commercial advantage and that copies bear this notice and the full citation on the first page. Copyrights for components of this work owned by others than ACM must be honored. Abstracting with credit is permitted. To copy otherwise, or republish, to post on servers or to redistribute to lists, requires prior specific permission and/or a fee. Request permissions from Permissions@acm.org.

ICONS '19, July 23-25, 2019, Knoxville, TN, USA

(C) 2019 Association for Computing Machinery.

ACM ISBN 978-1-4503-7680-8/19/07 .. \$15.00

https://doi.org/10.1145/3354265.3354272
}

\section{KEYWORDS}

Neuromorphic circuits, non-linear memristor model, radiation, spike-timing-dependent plasticity (STDP), leaky integrate-and-fire (LIF) neuron, spatio-temporal pattern learning

\section{INTRODUCTION}

Neuromorphic circuits or artificial neural networks are systems inspired by biological neural networks such as the brain. These highly connected networks process information in parallel and are widely used for pattern recognition tasks. Neural networks have recently been applied in areas including image processing for visual recognition [14], character recognition [5,23], voice-activated assistance [13], stock market forecasting [12], and self-driving cars [4].

In the near future, neuromorphic networks are likely to find application in harsh, radiation prone environments such as space and at nuclear and military installations. Currently, researchers are developing neural networks that could be used in solar radiation forecasting, large data capturing, object classification and matching, event filtering, facial recognition, combat automation, target identification and weapon optimization [9,17,20,26,27]. Thus, it is important to understand and model the effect of radiation events on neuromorphic circuits. While shielding and hardening are often used to protect devices and circuits, these techniques are unable to block all particles from interacting with underlying electronics $[10,16]$.

Multiple experimental studies have examined the effects of different types and intensity of radiation on memristive devices with different active materials and physical mode of operations $[3,7,8,11,19,22]$. This study uses the non-linear drift memristor model modified to capture its behavior under radiation [6]. The modified model is used in the simulation of a feed-forward memristor-based (many-to-one) neuromorphic circuit, which is taught to learn a spatio-temporal pattern by separating correlated and uncorrelated inputs. The circuit is exposed to state-altering radiation events of different flux, intensity, and duration during the learning period and the effect on the learning capacity of the network is observed. Section 2 briefly discusses the memristor model, neural network architecture, and its learning behavior. Section 3 discusses the effect of radiation on memristors and 
updated models, followed by section 4 that presents detailed network simulations with radiation effects. Section 5 concludes that once the radiation event ends, the neuromorphic circuit can resolve the pattern, but requires a longer time depending upon the radiation dose. Results also indicate that neuromorphic circuits have the capability to learn a pattern in the presence of low-flux and intensity radiation environment. This work shows that continuous training and use of the network can overcome larger amounts of radiation exposure. On the other hand, previous work showed that when the network is not undergoing training, the effects of radiation buildup and the deposited energy is not dissipated. The network therefore never achieves a stable state [6].

\section{NEUROMORPHIC CIRCUIT DESIGN}

\subsection{Non-Linear Drift Memristor Model}

The literature presents a wide range of memristor models $[1,18,25]$. Some of the models are more mathematical with many parameters to choose from making them input and application limited. This study uses the non-linear drift model as given by Chua et al. [2] which is motivated by $\mathrm{TiO}_{2}$ memristive devices. A window function is used to implement non-linearity. This model captures the non-linearity presented by memristive devices while using the physical characteristics of the device. Exact model parameters used in this study are presented in detail in [6]. The ratio $\mathrm{w} / \mathrm{D}$ is referred to as the state variable of the device at any given time and is bounded between maximum and minimum resistance $(0<\mathrm{w} / \mathrm{D}<1)$. The network and its components as in Figure 1 and Figure 2 were designed and simulated in the Cadence Virtuoso design suite using Verilog-A.

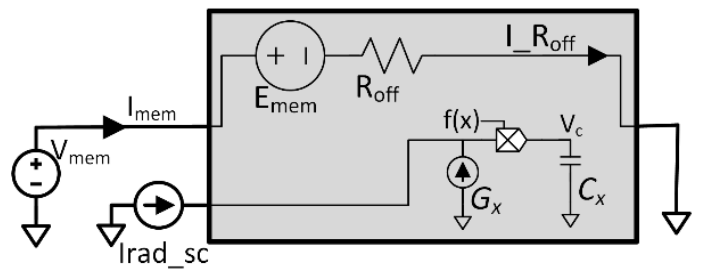

Figure 1: Non-linear drift memristor model implemented in Cadence Virtuoso Spectre. State-altering radiation (Irad_sc) is added to the auxiliary circuit so it can modify the state of device instantaneously.

\subsection{Pattern Learning and STDP}

Figure 3 shows the change in synaptic weight based on the relative timing of pre- and post-synaptic neuron (STDP learning curve) presented by all the memristors (M1 to M25) in the neural network during a $100 \mathrm{~s}$ pattern learning simulation. The conductance of the memristor increases when a pre-synaptic neuron (N1 to N25) spikes before the post-synaptic neuron and vice-versa. An interspike interval of up to $8 \mathrm{~ms}$ would affect the weight or conductivity (w/D) of the memristive device. This is also known as the learning window. Figure 3 inset also shows the triangular biphasic pre- and post-synaptic spikes of $10 \mathrm{~ms}$ used in the network.
The difference in the potentiation time of the two spikes leads to stronger depression in the STDP curve, which in turn leads to faster pattern learning.

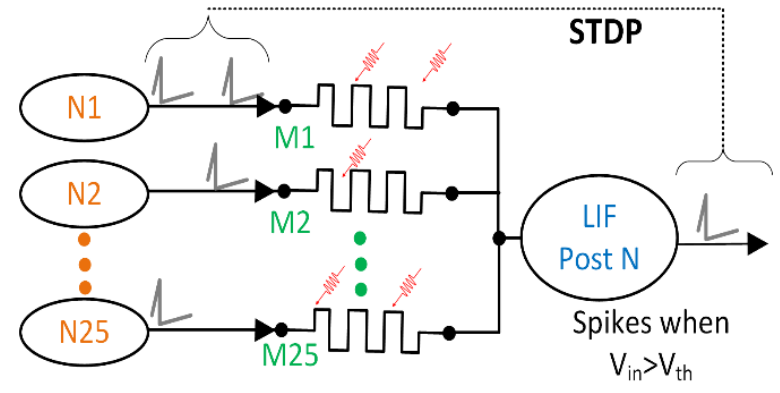

Figure 2: Memristor-based neural network used in this study. 25 pre-synaptic neurons are connected to one post-synaptic leaky integrate-and-fire neuron via single memristors. The network uses shaped pulses to achieve pair-based STDP for pattern learning.

In the neural network (Figure 2), a pre-synaptic neuron corresponding to white pixels in Figure 4 (b) fires mutually correlated spikes similar to ones in Figure 4 (a), N12 and N13. The remaining uncorrelated neurons (black pixels) fired uncorrelated, Poisson-distributed spikes similar to those in Figure 4 (a), N14 and N15. In other words, correlated afferents are simply firing periodically, whereas non-participating afferents fire randomly which could be produced through noise-generating circuits. All pre-synaptic neurons were firing at an average rate of $5 \mathrm{~Hz}$ [24]. Post-synaptic neuron spikes lead to a change in the state (w/D) of the memristors due to STDP.

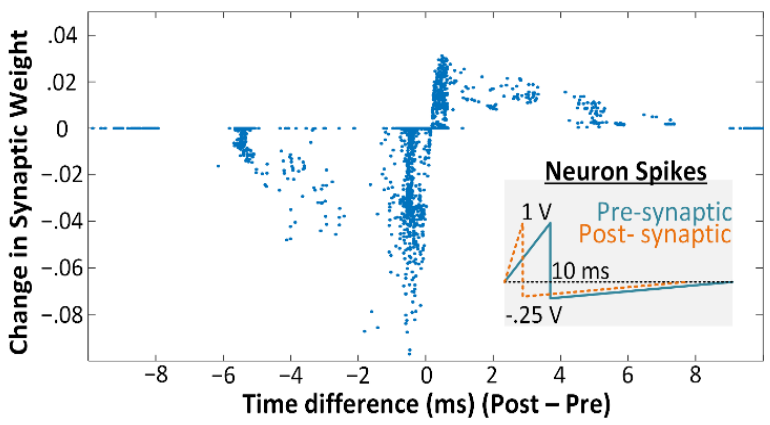

Figure 3: Change in synaptic weight based on the relative timing of pre- and post-synaptic action potentials. Weight change of all 25 synaptic devices in the neural network is plotted during $100 \mathrm{~s}$ of simulation. The inset shows the difference in pre- and post-synaptic neuron pulse shape that leads to fast learning and asymmetry in the STDP curve.

In this study, the network is learning a spatio-temporal pattern representing a 25-pixel character ' $\mathrm{B}$ '. All memristors were initially placed in low resistance as in Figure 4 (Initial State). After $30 \mathrm{~s}$ of learning, the network depressed the synaptic weight of uncorrelated (non-participating) neurons and the pattern is notable. At this point, the post-synaptic neuron is firing at a constant rate. 
(a) Input Neuron spiking pattern
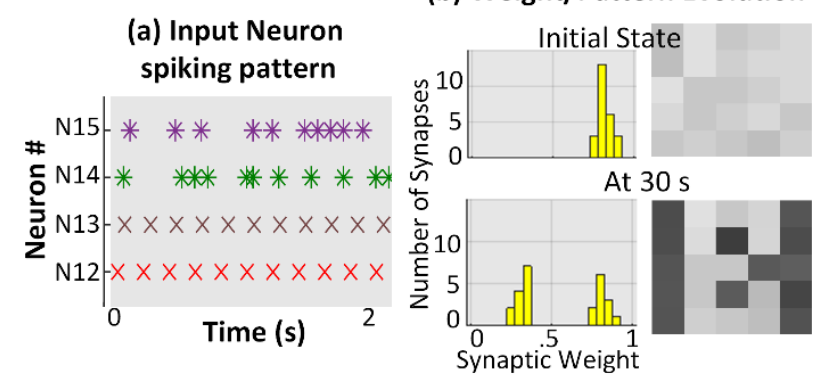

Figure 4: (a) Spike times of a few pre-synaptic neurons, two correlated (N12, N13) and two uncorrelated (N14, N15), firing at an average rate of $5 \mathrm{~Hz}$. (b) Change in synaptic weight of all the memristors. Initially (at $0 \mathrm{~s}$ ), memristors are kept in low resistance state. After $30 \mathrm{~s}$ of learning, the network was able to separate the uncorrelated synapses and the pattern starts to resolve.

\section{ADDING RADIATION}

\subsection{Modeling Radiation in Memristors}

Many factors influence the effect of a radiation event on a memristive device. A few of them are radiation trajectory, wavelength or energy of the radiation, secondary electron creation, and the thickness of the device shielding and packaging. Thus, in the literature, the experimental studies on memristor behavior in the presence of radiation leads to generally inconclusive results. However, $\mathrm{TiO}_{2}$ devices consistently show an increase in current (decrease in device resistance) after $\mathrm{x}$-ray, alpha or proton irradiation without affecting the high or low resistance limits of the devices $[8,19,22]$. As the device is bombarded by the radiation ions, more oxygen vacancies are generated thus instantaneously increasing the conductivity of the $\mathrm{TiO}_{2}$ layer. This paper focuses on modeling such widely observed state-altering $(\Delta \mathrm{w} / \mathrm{D})$ behavior in $\mathrm{TiO}_{2}$ memristive devices. Non-linear drift memristor model was modified to include the state change effect using radiation current as Irad_sc as in Figure 1, more details can be found in [6].

\subsection{Quantifying Radiation}

The radiation model used in [6] and in this study is essentially agnostic to the type of materials used in the memristor and the exact source of radiation. Radiation is artificially induced in the circuit using a current pulse of $1 \mathrm{~ms}$ duration. The current pulse directly changes the state of the memristive device in the model to make it more conductive. Radiation current pulse interval follows the random Poisson's distribution and magnitude follows random Gaussian distribution with mean $\mu$ and standard deviation $\sigma$. In this case, one current pulse does not necessarily correspond to one radiation interaction event. For example, 10, 20, 25, and 30 current pulses of magnitude $\mu=25 \mu \mathrm{A}$ and $\sigma=12.5 \mu \mathrm{A}$ induced $30 \%, 77 \%$, $90 \%$, and $95 \%$ change in resistance (from off state) of memristor in the model, respectively. Similar changes in resistance are experimentally observed in [7], [3,8], [21], and [19] using a total fluence of $7.7 \times 10^{15} 350-\mathrm{keV}$ proton $/ \mathrm{cm}^{2}, 1.4 \times 10^{11} 1-\mathrm{MeV}$ alpha $/ \mathrm{cm}^{2}, 4.9 \times 10^{12} 14.1-\mathrm{MeV}$ neutrons $/ \mathrm{cm}^{2}$, and $7.75 \times 10^{16} 10-$ $\mathrm{keV} \mathrm{x}$-rays $/ \mathrm{cm}^{2}$. These are experiments carried out under laboratory conditions using radiation sources of different intensities. Electronic circuits see a spectrum of radiation in space from ultraviolet and infrared to gamma and streams of electrons and protons during radiation flares. A spacecraft can observe from a few rad to $100 \mathrm{krad}(\mathrm{Si})$ around the trapped radiation belts like Jupiter [15]. The magnitude and frequency of the current pulses in the model were modulated by varying the mean current magnitude (following a random Gaussian distribution) and pulse interval (following a random Poisson distribution) [6]. As the mean magnitude and frequency (flux) of radiation current increases, the change in synaptic weight $(\Delta \mathrm{w} / \mathrm{D})$ of memristive device increases.

\subsection{Radiation Effects on STDP learning}

Figure 5 represents the change in the STDP curve at different radiation intensities. In this case, a radiation current pulse of a given intensity is induced in memristor before pre- and post-synaptic neuron spikes arrive, biphasic spikes used are as shown in Figure 5 (inset). It is noted as radiation intensity increased the memristors tended to favor a positive change in synaptic weight thus forcing artificial correlation. The shape of the STDP curve in Figure 5 is symmetrical because the pre- and post-synaptic spikes are the same. On the other hand, post-synaptic spikes in Figure 3 (and used in the network simulations) have stronger depression for reasons explained in section 2.2.

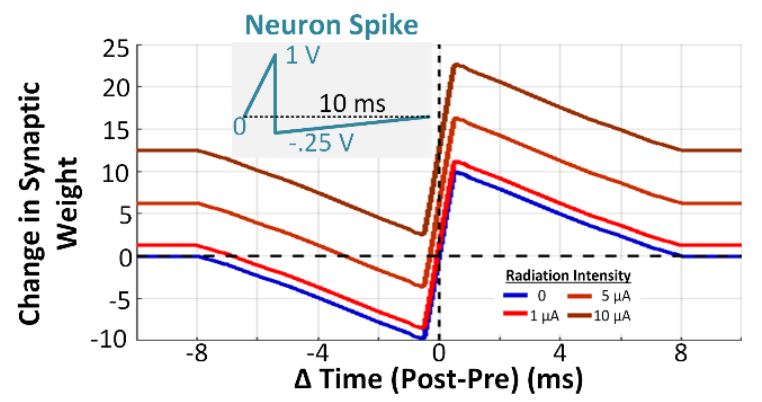

Figure 5: STDP pattern observed by memristor devices in the presence of radiation. Radiation brings asymmetry in the STDP curve favoring untrue correlation. The inset shows the shape of the pre- and post-afferent spikes used.

\section{RADIATION SIMULATIONS}

The following simulations present the learning behavior of the neural network when subjected to state-altering radiation of different flux, magnitude, and time. Initial conditions in each case were set the same as in Figure 4 (b). In sections 4.1 and 4.2, the system was able to resolve the pattern and separate uncorrelated synapses as in Figure 4 (b) (at $30 \mathrm{~s}$ ) and the radiation was induced 
in the network at this point (after $30 \mathrm{~s}$ ) for a specified time and/or flux intensity. In section 4.3, the system was irradiated continuously from $0 \mathrm{~s}$ until $100 \mathrm{~s}$ and the system's capability to learn the pattern in the presence of radiation is simulated. Current flux calculations are based on an assumed memristor device with interaction cross-section of $100 \mathrm{~nm}$ by $100 \mathrm{~nm}$.

\subsection{Effect of Radiation Flux on Learning}

Figure 6 represents the behavior of the neural network when irradiated with a flux of $10^{10} \mathrm{~cm}^{-2} \mathrm{~s}^{-1}$ (b), $3 \times 10^{10} \mathrm{~cm}^{-2} \mathrm{~s}^{-1}$ (c), and $5 \times 10^{10} \mathrm{~cm}^{-2} \mathrm{~s}^{-1}(\mathrm{~d})$ for $10 \mathrm{~s}$ (again, starting at $30 \mathrm{~s}$ ) at a magnitude $\mu=25 \mu \mathrm{A}$ and $\sigma=12.5 \mu \mathrm{A}$.

In Figure 6, the synaptic weight distribution is recorded right at the end of radiation events (at $40 \mathrm{~s}$ ) and at $70 \mathrm{~s}$ and $100 \mathrm{~s}$ as learning continued. It is observed that as flux increases to $5 \times 10^{10} \mathrm{~cm}^{-2} \mathrm{~s}^{-1}(\mathrm{~d})$ the pattern completely disappears (at $0 \mathrm{~s}$ post-radiation). As pattern learning continues after the end of radiation, the system was able to relearn the pattern even in the case of intense radiation flux (Figure 6 (d)). Although it did take much longer for the system to depress the non-participating afferents, the difference in synaptic weight distribution can be noted at $70 \mathrm{~s}$ and $100 \mathrm{~s}$.

(a) No radiation

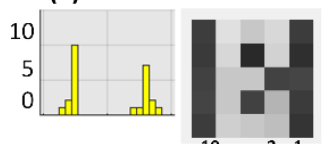

(b) Flux $1 \times 10^{10} \mathrm{~cm}^{-2} \mathrm{~s}^{-1}$
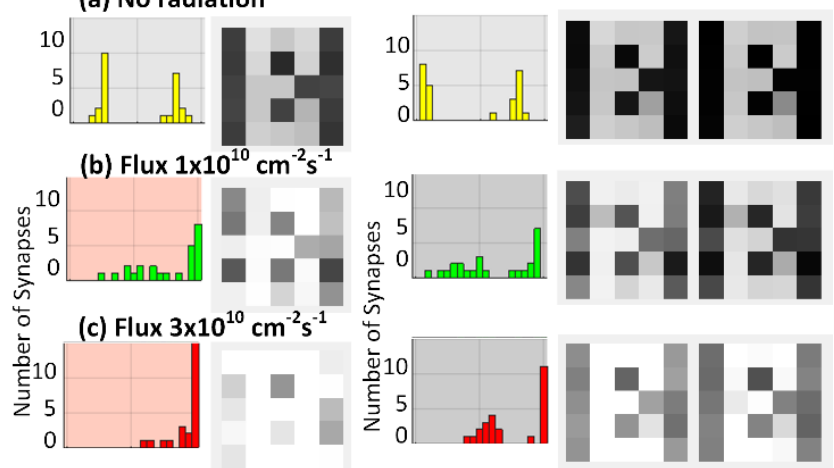

(d) Flux $5 \times 10^{10} \mathrm{~cm}^{-2} \mathrm{~s}^{-1}$
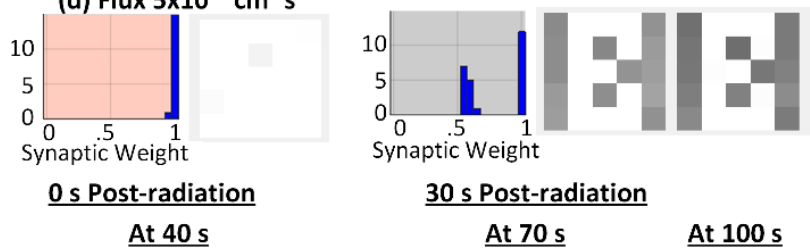

At $40 \mathrm{~s}$

Figure 6: Synaptic weight distribution immediately after the end of $10 \mathrm{~s}$ of radiation exposure (magnitude $\mu=25 \mu \mathrm{A}$ and $\sigma=$ $12.5 \mu \mathrm{A})$, at $70 \mathrm{~s}$, and $100 \mathrm{~s}$. As the flux increases, pattern distortion also increases, resulting in complete saturation in (d) at $40 \mathrm{~s}$. At the same time, the system was able to resolve the pattern and separate uncorrelated neurons but took much longer time to stabilize post-radiation.

Evolution of the average synaptic weight of all 25 memristors is plotted in Figure 7. Simulated irradiation of the system for $10 \mathrm{~s}$ starts at $30 \mathrm{~s}$ (grey region) at different flux with a mean magnitude $\mu=25 \mu \mathrm{A}$ and $\sigma=12.5 \mu \mathrm{A}$. As expected, after the end of radiation at $40 \mathrm{~s}$, mean weights start to evolve towards the non-radiated trace as the network tries to relearn the pattern. At higher flux of $5 \times 10^{10}$ $\mathrm{cm}^{-2} \mathrm{~s}^{-1}$, all weights saturate post-radiation and the network is unable to recognize the pattern as in (Figure 6 (d)) at $40 \mathrm{~s}$.

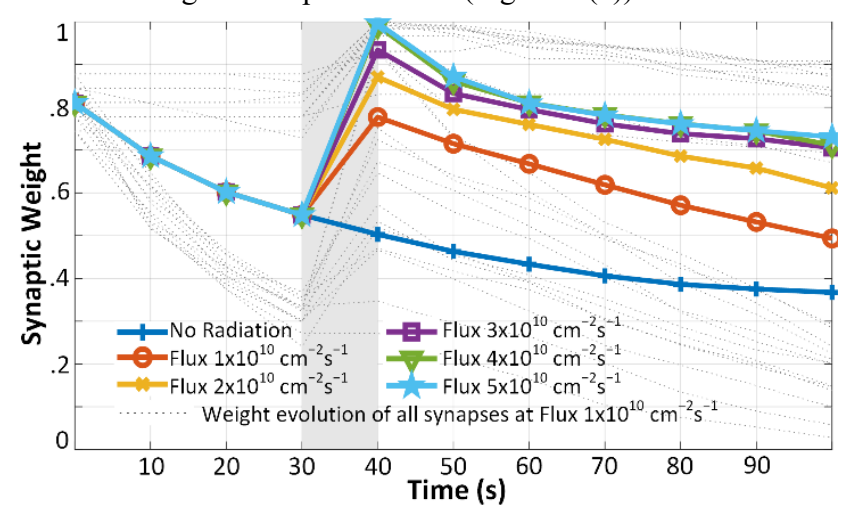

Figure 7: The average synaptic-weight evolution of all memristors as radiation flux increases. After $30 \mathrm{~s}$ of learning, memristors were simulated with state-altering radiation (magnitude $\mu=25 \mu \mathrm{A}$ and $\sigma=12.5 \mu \mathrm{A}$ ) for $10 \mathrm{~s}$ (grey area). After the end of the radiation event as the network tries to relearn the pattern, the average synaptic weight of radiated memristors evolves towards the non-radiated weight curve.

\subsection{Effect of Radiation Duration on Learning}

Figure $8 \mathrm{~b}$ and $\mathrm{c}$ represent the behavior of the neural network when irradiated for a longer period $(20 \mathrm{~s}$ and $40 \mathrm{~s}$, starting at time 30 s) with Gaussian distributed pulses of average magnitude $\mu=5$ $\mu \mathrm{A}$ and $\sigma=2.5 \mu \mathrm{A}$ at $3 \times 10^{10} \mathrm{~cm}^{-2} \mathrm{~s}^{-1}$ flux.

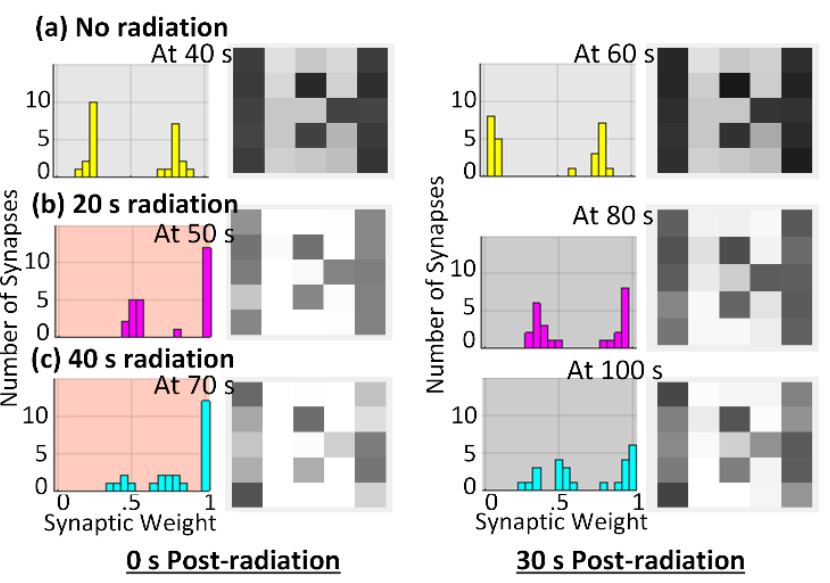

Figure 8: The left column shows the synaptic weight distribution after the end of state-altering radiation event $\left(3 \times 10^{10} \mathrm{~cm}^{-2} \mathrm{~s}^{-1}\right.$ flux, magnitude $\mu=5 \mu \mathrm{A}$ and $\left.\sigma=2.5 \mu \mathrm{A}\right)$ for (b) $20 \mathrm{~s}$ and (c) $40 \mathrm{~s}$, after $30 \mathrm{~s}$ of uninterrupted learning. The right column shows the weight distribution $30 \mathrm{~s}$ after the end of radiation. In (c), the network is still in an early stage of learning as radiation effects accumulated over time and delay the learning process.

The weight distribution was recorded right at the end of radiation events (at $50 \mathrm{~s} / 70 \mathrm{~s}$ ) and after $30 \mathrm{~s}$ (at $80 \mathrm{~s} / 100 \mathrm{~s}$ ). Figure 
6 (d) at $40 \mathrm{~s}$ shows more distortion in the pattern than Figure 8 (c) at $70 \mathrm{~s}$ even though Figure 8 (c) saw a longer period of exposure that is due to lower flux. Thus, it was also quicker for the network to depress the neurons and relearn the pattern.

Figure 9 plots the evolution of average synaptic weight when the system is irradiated for longer periods (colored region) with pulse magnitude $\mu=5 \mu \mathrm{A}$ and $\sigma=2.5 \mu \mathrm{A}$ each at $3 \times 10^{10} \mathrm{~cm}^{-2} \mathrm{~s}^{-1}$ flux. After the end of the radiation events, mean weights start to evolve towards the non-irradiated trace. Even when exposed to radiation for $40 \mathrm{~s}$, weights do not saturate unlike the effect observed in Figure 7 at higher flux.

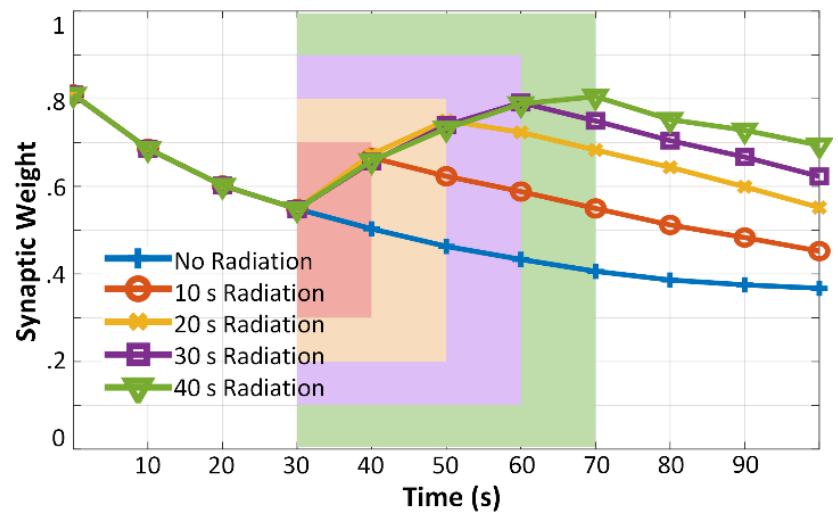

Figure 9: Average synaptic-weight evolution of all memristors as state-altering radiation $\left(3 \times 10^{10} \mathrm{~cm}^{-2} \mathrm{~s}^{-1}\right.$ flux, magnitude $\mu=$ $5 \mu \mathrm{A}$ and $\sigma=2.5 \mu \mathrm{A}$ ) time increases from $10 \mathrm{~s}$ to $40 \mathrm{~s}$ (colored area). After the end of the radiation event as the network tries to relearn the pattern, the average synaptic weight of radiated memristors evolves towards the non-radiated weight curve.

\subsection{Learning During Radiation}

Simulations results shown in Figure 10 and Figure 11 demonstrate the learning ability and the average synaptic weight evolution of the network in the presence of radiation of pulse magnitude $\mu=.5 \mu \mathrm{A}$ and $\sigma=.25 \mu \mathrm{A}$ at different flux. In these cases, radiation events started at $0 \mathrm{~s}$ when the network was in its initial state as in Figure 4 (b). The goal of this experiment was to determine if the network can learn a pattern at all in the presence of radiation, or whether the weight evolutions are inevitably altered.

Figure 10 shows until $40 \mathrm{~s}$ there was no major disruption in the network's ability to learn the pattern. Figure 10 (b) at a lower flux of $1 \times 10^{10} \mathrm{~cm}^{-2} \mathrm{~s}^{-1}$ shows no change in pattern recognition capability of the network for $100 \mathrm{~s}$. It can be observed from Figure 10 (c, d, and e) that as radiation flux increases network quickly becomes unstable sooner as radiation accumulates. A similar evolution can be noted in Figure 11, which plots the total average weight of all the afferent synapses versus time. In this plot, weight evolution is similar until flux reaches over $1 \times 10^{10} \mathrm{~cm}^{-2} \mathrm{~s}^{-1}$. Here, the flux weight evolution is similar to the no radiation curve, but higher flux causes a sudden decrease in total weight after $70 \mathrm{~s}, 50 \mathrm{~s}$ and $40 \mathrm{~s}$ in case of
$3 \times 10^{10} \mathrm{~cm}^{-2} \mathrm{~s}^{-1}, 4 \times 10^{10} \mathrm{~cm}^{-2} \mathrm{~s}^{-1}$, and $5 \times 10^{10} \mathrm{~cm}^{-2} \mathrm{~s}^{-1}$ state-altering radiation flux.

(a) No radiation
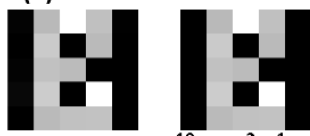

(b) Flux $1 \times 10^{10} \mathrm{~cm}^{-2} \mathrm{~s}^{-1}$

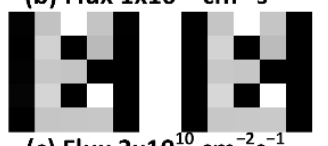

(c) Flux $3 \times 10^{10} \mathrm{~cm}^{-2} \mathrm{~s}^{-1}$
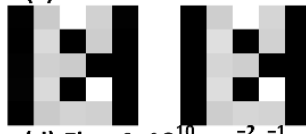

(d) Flux $4 \times 10^{10} \mathrm{~cm}^{-2} \mathrm{~s}^{-1}$

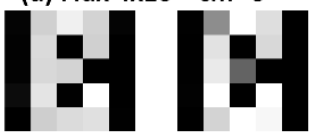

(e) Flux $5 \times 10^{10} \mathrm{~cm}^{-2} \mathrm{~s}^{-1}$
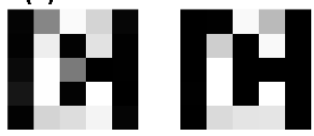

At $40 \mathrm{~s}$
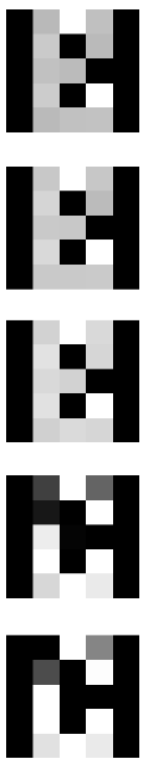

At $60 \mathrm{~s}$
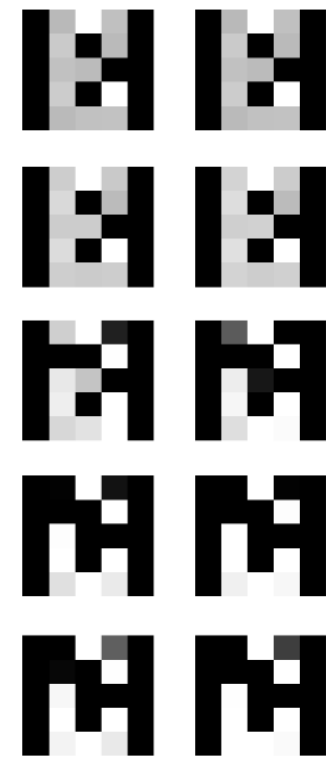

At $80 \mathrm{~s}$

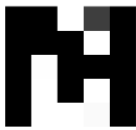

At $100 \mathrm{~s}$

Figure 10: Memristors were exposed to state-altering radiation (flux magnitude $\mu=.5 \mu \mathrm{A}$ and $\sigma=.25 \mu \mathrm{A}$ ) throughout the learning process (for $100 \mathrm{~s}$ starting at $0 \mathrm{~s}$ ). In each case network was able to resolve the pattern in $40 \mathrm{~s}$. Although, at higher flux (c), (d) and (e) the network became unstable at $80 \mathrm{~s}$, $60 \mathrm{~s}$, and 50 s. Network maintained the stability in (b) at lower flux value.

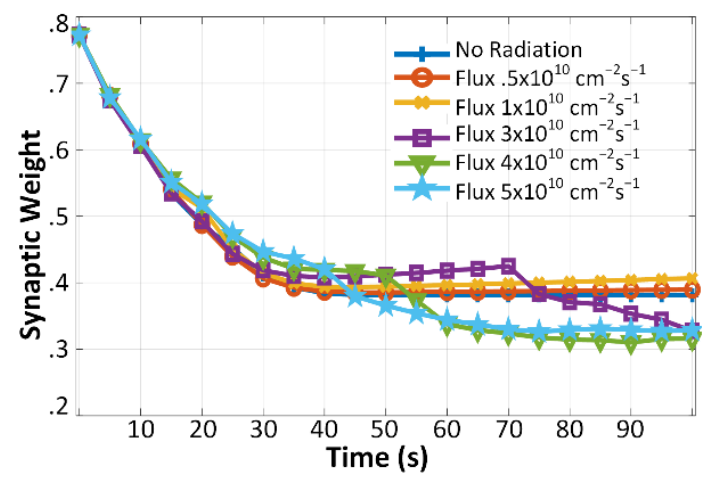

Figure 11: Average synaptic-weight evolution of all memristors as the network tries to learn the pattern in presence of statealtering radiation (for $100 \mathrm{~s}$ starting at $0 \mathrm{~s}$ ) at different flux (pulse magnitude with $\mu=.5 \mu \mathrm{A}$ and $\sigma=.25 \mu \mathrm{A}$ ). The network tries to resolve the pattern but becomes unstable sooner as the flux increases but at lower flux network was successfully able to recognize the pattern throughout the time. 
It is interesting to note that in both Figure 10 ( $\mathrm{d}$ and e), the network becomes stable again at $80 \mathrm{~s}$, but consistently starts recognizing a different pattern. Figure 11 shows the stable evolution of weight after $70 \mathrm{~s}$ in both cases when the flux is at $4 \times 10^{10} \mathrm{~cm}^{-2} \mathrm{~s}^{-1}$, and $5 \times 10^{10} \mathrm{~cm}^{-2} \mathrm{~s}^{-1}$. More exploration and longer simulations are required to understand the behavior and stability of the network under these conditions.

\section{CONCLUSIONS}

The effect of state-altering radiation on a memristor-based neuromorphic circuit was examined. The circuit learns spatiotemporal patterns by correlated spike timing and STDP. Subject to continued training, the network starts to recover when exposed to short-term radiation that alters the synaptic states. Since the memristive devices have to cope with the effect of radiation and relearn the pattern, system-learning time increased based on flux, intensity, and length of irradiation. The network is able to learn and separate the uncorrelated afferents when the pattern was presented in low flux radiation.

At the same time, higher radiation flux causes instability in the network. To gain stability over time, it suppresses the inputs from a few of the correlated neurons and seems to learn a new pattern. Thus, future work includes analyzing the long-term effects of radiation on the pattern learning capability of the network. It may also be useful to incorporate features such as recurrent connections and inhibitory synapses, which may increase the stability of the network. Although the network used in this study are two-layer networks with relatively few neurons, they represent a part of multi-layer deep spiking neural network where convolution layers may be able to recover from the insignificant errors at lower radiation exposures.

\section{ACKNOWLEDGMENTS}

This work was supported by the Defense Threat Reduction Agency (DTRA) grant HDTRA1-17-1-0036.

\section{REFERENCES}

[1] Hisham Abdalla and Matthew D. Pickett. 2011. SPICE modeling of memristors. Proc. - IEEE Int. Symp. Circuits Syst. (2011), 1832-1835. DOI:https://doi.org/10.1109/ISCAS.2011.5937942

[2] Shyam Prasad Adhikari, Changju Yang, Hyongsuk Kim, and Leon O. Chua. 2012. Memristor bridge synapse-based neural network and its learning. IEEE Trans. Neural Networks Learn. $\begin{array}{lllll}\text { Syst. } & 23, & 9 & \text { (2012), } & 1426-1435 .\end{array}$ DOI:https://doi.org/10.1109/TNNLS.2012.2204770

[3] H. J. Barnaby, S. Malley, M. Land, S. Charnicki, A. Kathuria, B. Wilkens, E. Deionno, and W. M. Tong. 2011. Impact of alpha particles on the electrical characteristics of TiO 2 memristors. IEEE Trans. Nucl. Sci. 58, 6 PART 1 (2011), 2838-2844. DOI:https://doi.org/10.1109/TNS.2011.2168827

[4] Mariusz Bojarski, Davide Del Testa, Daniel Dworakowski, Bernhard Firner, Beat Flepp, Prasoon Goyal, Lawrence D. Jackel, Mathew Monfort, Urs Muller, Jiakai Zhang, Xin Zhang, Jake Zhao, and Karol Zieba. 2016. End to End Learning for SelfDriving Cars. (April 2016). Retrieved January 18, 2019 from https://arxiv.org/pdf/1604.07316.pdf

[5] Dan Claudiu Ciresan, Ueli Meier, Luca Maria Gambardella, and Jürgen Schmidhuber. 2010. Deep, Big, Simple Neural Nets for
Handwritten Digit Recognition. Neural Comput. 22, 12 (December 2010), 3207-3220. DOI:https://doi.org/10.1162/NECO_a_00052

[6] Sumedha Gandharava Dahl, Robert Ivans, and Kurtis D. Cantley. 2018. Modeling Memristor Radiation Interaction Events and the Effect on Neuromorphic Learning Circuits. In Proceedings of the International Conference on Neuromorphic Systems - ICONS '18, 1-8. DOI:https://doi.org/10.1145/3229884.3229885

[7] E. Deionno, M. D. Looper, J. V. Osborn, and J. W. Palko. 2013. Displacement damage in Tio2 Memristor devices. IEEE Trans. Nucl. $\quad$ Sci. $\quad 60, \quad 2 \quad$ (2013), 1379-1383. DOI:https://doi.org/10.1109/TNS.2013.2249529

[8] Erica Deionno, Mark D. Looper, Jon V. Osborn, Hugh J. Barnaby, and William M. Tong. 2013. Radiation effects studies on thin film TiO2 memristor devices. IEEE Aerosp. Conf. Proc (2013), 1-8. DOI:https://doi.org/10.1109/AERO.2013.6497378

[9] A Dmitriev, Yu Minaeva, Yu Orlov, I Persiantsev, A Suvorova, and I Veselovsky. ARTIFICIAL NEURAL NETWORK APPLICATIONS TO THE SPACE RADIATION ENVIRONMENT MODELLING AND FORECASTING. Retrieved March 8, 2019 from http://dec1.npi.msu.su/english/data/lasre/index.html

[10] P. E. Dodd, M. R. Shaneyfelt, J. R. Schwank, and J. A. Felix. 2010. Current and future challenges in radiation effects on CMOS electronics. IEEE Trans. Nucl. Sci. 57, 4 PART 1 (2010), 17471763. DOI:https://doi.org/10.1109/TNS.2010.2042613

[11] Yago Gonzalez-Velo, Hugh J. Barnaby, and Michael N. Kozicki. 2017. Review of radiation effects on ReRAM devices and technology. Semicond. Sci. Technol. 32, 8 (2017). DOI:https://doi.org/10.1088/1361-6641/aa6124

[12] Zabir Haider Khan, Tasnim Sharmin Alin, and Akter Hussain. 2011. Price Prediction of Share Market Using Artificial Neural Network “ANN." Int. J. Comput. Appl. 22, 2 (May 2011), 42-47. DOI:https://doi.org/10.5120/2552-3497

[13] Geoffrey Hinton, Li Deng, Dong Yu, George Dahl, Abdelrahman Mohamed, Navdeep Jaitly, Andrew Senior, Vincent Vanhoucke, Patrick Nguyen, Tara Sainath, and Brian Kingsbury. 2012. Deep Neural Networks for Acoustic Modeling in Speech Recognition: The Shared Views of Four Research Groups. IEEE Signal Process. Mag. 29, 6 (November 2012), 82-97. DOI:https://doi.org/10.1109/MSP.2012.2205597

[14] Shuiwang Ji, Wei Xu, Ming Yang, and Kai Yu. 2013. 3D Convolutional Neural Networks for Human Action Recognition. IEEE Trans. Pattern Anal. Mach. Intell. 35, 1 (January 2013), 221-231. DOI:https://doi.org/10.1109/TPAMI.2012.59

[15] A.H. Johnston and S.M. Guertin. The effects of space radiation on linear integrated circuits. In 2000 IEEE Aerospace Conference. Proceedings (Cat. No.00TH8484), 363-369. DOI:https://doi.org/10.1109/AERO.2000.878509

[16] Andrew S. Keys, James H. Adams, John D. Cressler, Ronald C. Darty, Michael A. Johnson, and Marshall C. Patrick. 2008. Highperformance, radiation-hardened electronics for space and lunar environments. AIP Conf. Proc. 969, (2008), 749-756. DOI:https://doi.org/10.1063/1.2845040

[17] V. Sampath Kumar, J. Prasad, V. Lakshmi Narasimhan, and S. Ravi. 2017. Application of artificial neural networks for prediction of solar radiation for Botswana. In 2017 International Conference on Energy, Communication, Data Analytics and Soft Computing (ICECDS), r 3493-3501. DOI:https://doi.org/10.1109/ICECDS.2017.8390110

[18] Shahar Kvatinsky, Eby G. Friedman, Avinoam Kolodny, and Uri C. Weiser. 2013. TEAM: Threshold adaptive memristor model IEEE Trans. Circuits Syst. I Regul. Pap. 60, 1 (2013), 211-221. DOI:https://doi.org/10.1109/TCSI.2012.2215714

[19] Matthew J Marinella, Scott M Dalton, Patrick R Mickel, Paul E Dodd Dodd, Marty R Shaneyfelt, Edward Bielejec, Gyorgy Vizkelethy, and Paul G Kotula. 2012. Initial Assessment of the Effects of Radiation on the Electrical Characteristics of Memristive Memories. Nucl. Sci. IEEE Trans. 59, 6 (2012), 2987-2994. DOI:https://doi.org/10.1109/TNS.2012.2224377 
[20] T.V. Santosh, Gopika Vinod, R.K. Saraf, A.K. Ghosh, and H.S. Kushwaha. 2007. Application of artificial neural networks to nuclear power plant transient diagnosis. Reliab. Eng. Syst. Saf. 92,
10
(October
2007),
1468-1472.

DOI:https://doi.org/10.1016/J.RESS.2006.10.009

[21] J. L. Taggart, R. Fang, Y. Gonzalez-Velo, H. J. Barnaby, M. N. Kozicki, N. Chamele, A. Mahmud, and M. Mitkova. 2016. Effects of $14 \mathrm{MeV}$ neutron irradiation on the DC characteristics of CBRAM cells. 2016 16th Eur. Conf. Radiat. Its Eff. Components Syst. (September 2016), 1-4. DOI:https://doi.org/10.1109/RADECS.2016.8093120

[22] William M. Tong, J. Joshua Yang, Philip J. Kuekes, Duncan R. Stewart, R. Stanley Williams, Erica DeIonno, Everett E. King, Steven C. Witczak, Mark D. Looper, and Jon V. Osborn. 2010. Radiation hardness of $\mathrm{TiO} 2$ memristive junctions. IEEE Trans. Nucl. Sci. 57, 3 PART 3 (2010), 1640-1643. DOI:https://doi.org/10.1109/TNS.2010.2045768

[23] Ruthvik Vaila, John Chiasson, and Vishal Saxena. 2019. Deep Convolutional Spiking Neural Networks for Image Classification. (March 2019). Retrieved June 25, 2019 from http://arxiv.org/abs/1903.12272

[24] Stanislaw Wozniak, Tomas Tuma, Angeliki Pantazi, and Evangelos Eleftheriou. 2016. Learning spatio-temporal patterns in the presence of input noise using phase-change memristors. Proc. - IEEE Int. Symp. Circuits Syst. 2016-July, (May 2016), 365-368. DOI:https://doi.org/10.1109/ISCAS.2016.7527246

[25] Chris Yakopcic, Tarek M. Taha, Guru Subramanyam, and Robinson E. Pino. 2013. Generalized memristive device SPICE model and its application in circuit design. IEEE Trans. Comput. Des. Integr. Circuits Syst. 32, 8 (2013), 1201-1214. DOI:https://doi.org/10.1109/TCAD.2013.2252057

[26] Artificial Intelligence and the Military | RealClearDefense. Retrieved March 8, 2019 from https://www.realcleardefense.com/articles/2017/09/07/artificial intelligence and the military $112240 \mathrm{html}$

[27] Kalashnikov develops fully automated neural network-based combat module - RT World News. Retrieved March 8, 2019 from https://www.rt.com/news/395375-kalashnikov-automatedneural-network-gun 